UNIVERSITÀ CATTOLICA DEL SACRO CUORE

Dipartimento di Economia e Finanza

\author{
Working Paper Series
}

An empirical analysis of the global inputoutput network and its evolution

Jakob Grazzini, Alessandro Spelta

Working Paper n. 31

October 2015

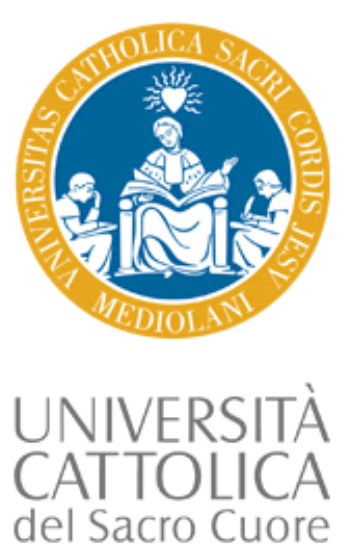




\title{
An empirical analysis of the global input- output network and its evolution
}

\author{
Jakob Grazzini \\ Università Cattolica del Sacro Cuore
}

Alessandro Spelta

Università Cattolica del Sacro Cuore

\section{Working Paper n. 31}

October 2015

\author{
Dipartimento di Economia e Finanza \\ Università Cattolica del Sacro Cuore \\ Largo Gemelli 1 - 20123 Milano - Italy \\ tel: +39.02.7234.2976 - fax: +39.02.7234.2781 \\ e-mail: dip.economiaefinanza@unicatt.it
}

The Working Paper Series promotes the circulation of research results produced by the members and affiliates of the Dipartimento di Economia e Finanza, with the aim of encouraging their dissemination and discussion. Results may be in a preliminary or advanced stage. The Dipartimento di Economia e Finanza is part of the Dipartimenti e Istituti di Scienze Economiche (DISCE) of the Università Cattolica del Sacro Cuore. 


\title{
An empirical analysis of the global input-output network and its
}

\section{evolution}

\author{
Jakob Grazzini*a,b and Alessandro Spelta ${ }^{\dagger a, b}$ \\ ${ }^{\mathrm{a}}$ Catholic University of Milan, Department of Economics and Finance, via Necchi 5, 20123, Milano, Italy. \\ ${ }^{\mathrm{b}}$ Complexity Lab in Economics, Milano, Italy
}

October 2015

\begin{abstract}
Data, without a model, are just numbers. The analysis of networks in economics should take into account how economic agents react to exogenous shocks. In order to determine the centrality of a node in the global input-output network, we analyze the network through the lenses of an economic model calibrated on empirical data. We show that formalizing the nodes as firms, and modeling the links as the result of firms' behavior (trade), is important for the economic interpretation of the network topology. Moreover, using the calibrated model, we define a fragility index that measures the ability of the system to absorb exogenous shocks. We find that the fragility of the production network has increased from 1995 to 2011.
\end{abstract}

Keywords: Input-output, Network Analysis

JEL codes: D57, D85, L14

\section{Introduction}

The importance of firms' production network in affecting micro and macro economic behavior has been stressed by past catastrophic events (Carvalho, 2014):

When flood waters rose in Thailand, Wal-Mart stores in Japan ran out of mouthwash(...). A string of natural disasters has exposed a vulnerability in global supply

\footnotetext{
${ }^{*}$ Email: jakob.grazzini@unicatt.it

${ }^{\dagger}$ Email: alessandro.spelta@unicatt.it
} 
chains. How do you set up a network that is compact enough to be efficient but spreads widely enough so that no single unexpected event can knock it out? Japan's earthquake and tsunami in March caused auto parts shortages worldwide. Thailand's recent flooding shut down some of the world's largest hard drive makers, which could cut personal computer shipments by as much as 20 percent in the first quarter of 2012. (Reuters 2011) ${ }^{1}$

Similarly, during the recent financial crisis, there has been much discussion about firms that are too big to fail. As reported by Carvalho (2014), the bail-out of General Motors was perceived as necessary to avoid disruptions in the supply chain of the American automotive industry. The idea that sectoral interdependencies, arising from the input-output structure, has an important influence on aggregate economic behavior has a long history in economics. More recently it has been revived by Long Jr and Plosser (1983). Horvath (1998, 2000) and Acemoglu et al. (2012), show that the topology of the input-output network has a crucial role in determining the aggregate behavior of the system. If the input-output network is significantly asymmetric, i.e. if relatively few sectors play a predominant role as suppliers, then idiosyncratic shocks give rise to aggregate fluctuations. When the organization of production is dominated by a small number of hubs supplying inputs to many different sectors, disruptions in these critical nodes can affect the global production system, determining losses in production and welfare (Acemoglu et al., 2012). Understanding the structure of the production network, and in particular determining which sectors act as hubs in the network, is important to understand the origin of aggregate fluctuations and to inform policymakers on how to prepare for, and recover from, adverse shocks hitting the production network.

In complex network theory, these key sectors are identified by applying appropriate measures of node centrality. The idea of centrality was initially proposed in the context of social systems, where a relation between the location of a subject in the social network and its influence on group processes was assumed. Various measures of centrality have been proposed in network theory such as the number of neighbors of a node (degree centrality), which is a local centrality measure, or measures based on the spectral properties of the graph (see Perra and Fortunato, 2008). Spectral centrality measures include the eigenvector centrality (Bonacich, 1972; Bonacich and Lloyd, 2001), Katz's centrality (Katz, 1953), PageRank (Brin and Page,

\footnotetext{
${ }^{1}$ Retrieved on 26 March 2015 from http://www.reuters.com/article/2011/11/13/us-apec-disasteridUSTRE7AC12Q20111113
} 
1998), hub and authority centralities (Kleinberg, 1999). Those measures are feedback (global) centrality measures and provide information on the position of each node relative to all other nodes.

Centrality analysis has been recognized to provide important insights into economic phenomena. For example, Kali and Reyes $(2007,2010)$ studied how the position in the trade network, specified by the number of links of a country (node degree), has substantial implications for economic growth and a good potential in predicting episodes of financial contagion. Schiavo et al. (2010) showed that node centrality measures, such as the degree/strength and the random betweenness centrality of the nodes, applied to trade and financial networks, may help to account for the evolution of international economic integration. Chinazzi et al. (2013) showed that econometric models, fed with international financial network measures, provide useful information about the aggregate performance of different countries.

In this paper, we link the empirical study of the global input-output network with the insights provided by an economic model. In order to determine the centrality of a node in the input-output network, we analyze the network through the lenses of an economic model that is calibrated on empirical data. We build a model along the lines of Long Jr and Plosser (1983) and Acemoglu et al. (2012), and we calibrate the technological parameters and normalize the nominal wage so that the model reproduces the observed nominal flows of trade. This means that each firm in the model produces the same output, employs the same inputs, and has the same links as the observed counterpart.

We claim that it is not enough to use only the information contained in the topological measures of the observed network. To understand the importance of an industry in the world input-output network it is necessary to employ a model to properly formalize links and nodes in economic terms. A node, in our context, is a firm which represents an industry or sector whose maximizing behavior determines quantities and prices. A link is a trade relationship between two sectors explained by technological and behavioral factors. The model gives a theoretical structure to the input-output network, and in particular it gives a precise economic meaning to shocks and to their spreading. A shock to node $i$ is defined as a productivity shock to industry $i$. The spreading of the shock is measured by the effect on production costs and prices to all other industries in the network. The empirical input-output network, on the other hand, gives us the path over which the shock propagates between sectors.

Using the calibrated model it is possible to build a network of effects, where the nodes 
are the sectors and the links represent the direct and indirect effect of a shock to a node on production costs of all other nodes. The average out-strength of a node in the network of effects therefore represents the influence of that node on the global production costs. We will call this measure cost effect. The assumptions about the production and utility functions and about agents' behavior allow also to define a measure for the real GDP and to investigate the effects of a shock to each sector on the world real GDP. We call this measure GDP effect.

Using the cost effect and the GDP effect we study the properties of the global input-output network in 2011, and its evolution from 1995 to 2011. We find that the influence of the different sectors on the global production is highly asymmetrical, i.e. few sectors have very strong impact on the global system. Moreover we find that between 1995 and 2011, the fragility of the input-output system increased due to a change in the production organization and that some peripheral sectors in 1995 became very important in 2011.

The contribution of this paper is to use an empirically calibrated model to analyze the complex network of relations in global production, determine the most influential sectors and understand the evolution of the network from 1995 to 2011.

The paper is organized as follows. In section 2 we analyze the topological characteristics of the world input-output network in 2011. In section 3, we describe a simple economic model with an input-output structure. The model is calibrated to the WIOT 2011 data. To analyze the influence of the sectors in global production we define and analyze a cost effect index (in section 4) and a GDP effect index (in section 5). In section 6, we exploit the yearly WIOT data from 1995 to 2011, to explore how the overall network fragility and how the influence of sectors evolve over time. Section 7 concludes.

\section{The empirical world input-output network}

\section{$2.1 \quad$ The data}

The World Input-Output Table (WIOT) is a database providing the input-output tables for 40 countries $^{2}$ and 35 industries and a set of final consumption sectors for each country, covering the period from 1995 to 2011 (Timmer et al., 2012). The input-output table records the nominal flows of goods between each sector in each country, at current prices. We aggregate the final consumption sectors in one global sector. ${ }^{3}$

\footnotetext{
${ }^{2} 27$ EU countries and 13 other major countries in the world and a model for the rest of the world.

${ }^{3}$ For further details on the data, see Timmer et al. (2012) and appendix B
} 


\subsection{Empirical analysis of the WIOT network}

The WIOT has a network representation in which a generic element $W_{i, j}$ represents the nominal amount of goods $j$ used as input by sector $i$, with $i, j=1, \ldots, N$, where $N$ is the number of sectors. The nodes are sectors and the links are the nominal flow of goods between sectors ${ }^{4}$. The WIOT network is therefore a directed and weighted network. In order to understand the importance of a node in the global production system, the literature on networks provides a set of centrality measures. The simplest measure is the degree centrality, which is defined as the total number of links attached to a node. Since the input-output network is directed and weighted, we use the directed-weighted version of degree centrality. We compute the weighted in-degree (or in-strength) and the weighted out-degree (out-strength) of each node. The instrength is the sum of all incoming flows of goods, i.e. the sum of the nominal inputs used by a sector. The out-strength is the sum of the outflow of goods, i.e. the sum of the nominal inputs produced by a sector. Formally:

$$
k_{i n, i}^{w}=\sum_{j=1}^{N} W_{i, j} \quad k_{\text {out }, i}^{w}=\sum_{j=1}^{N} W_{j, i}
$$

where $k_{i n, i}^{w}$ is the in-strength of node $i$ (i.e. the sum of elements of row $i$ ), and $k_{o u t, i}^{w}$ is the out-strength of node $i$ (i.e. the sum of elements of column $i$ ). The degree centrality is a local measure of centrality, because it does not include information about the network position of the neighbors of a node, nor about the network position of the neighbors of its neighbors, etc. A well-known global centrality measure is the core of Google's PageRank algorithm (Brin and Page, 1998). The idea behind PageRank is that a node is systemically important if its neighbors are important and/or the neighbors of the neighbors are important (for a formal definition of the PageRank see appendix A).

In figure 1 we show the tail distributions ${ }^{5}$ of nodes' in-strength and out-strength scores (left panel) and nodes' input PageRank and output PageRank (central panel ). To have a complete topological description of the flow of goods in the world production network, we analyze the share of each sector in global consumption. We define $c_{i}$ as the share of global nominal GDP consumed in each sector, and show its tail distribution in the right panel of figure 1 . In order

\footnotetext{
${ }^{4}$ In order to analyze the WIOT network we look only at the flows of goods between industries, ignoring the flows of goods toward the final consumption sector. In this way we obtain a quadratic matrix. The consumption vector will be used to calibrate the utility function in the model.

${ }^{5}$ The tail distribution of $x$ is defined as the probability $P(x>\bar{x})$.
} 

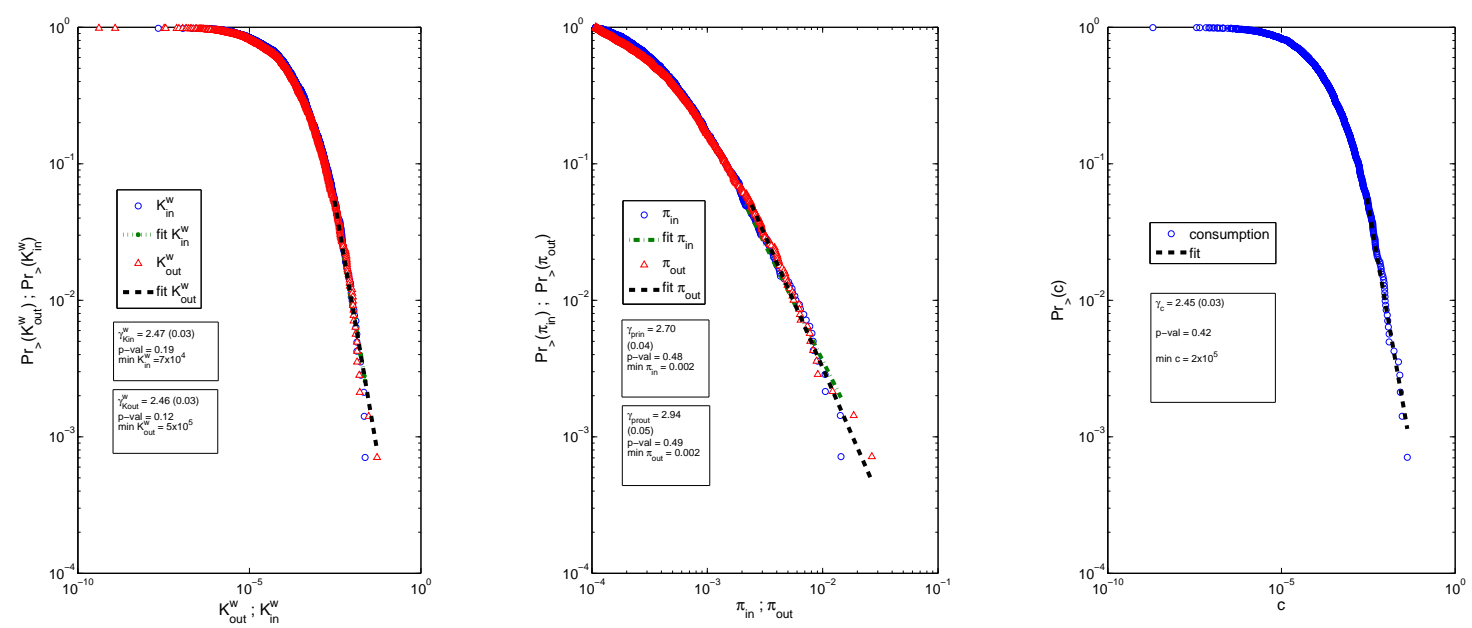

Figure 1: Log-log plot of tail distribution functions and maximum likelihood power-law fits of the in- and outstrength distributions (Left), of the in- and out-PageRank (Center), and of consumption distribution (Right). In the boxes we report the value of the estimated exponents, the p-values and the $x_{m i n}$ parameter characterizing the estimated distributions. The parameter $x_{\min }$ is the percenti

to further characterize the tail distributions, we test whether the empirical distributions are consistent with the hypothesis that they are drawn from a power law distribution. A power law distribution in general is defined as:

$$
P(x>\bar{x}) \propto\left(\frac{x}{x_{\min }}\right)^{-\gamma}
$$

We estimate the parameters $\gamma$ and $x_{\min }$ for each empirical distribution and test for power law hypothesis following Clauset et al. (2009). The parameter $\gamma$ is the exponent or scaling parameter (Clauset et al., 2009), and $x_{m i n}$ determines the minimum value after which the distribution of $x$ follows a power-law. ${ }^{6}$ The results of the estimation and test procedure are shown in figure 1. According to Clauset et al. (2009) the test can not be rejected if the p-value (reported in figure 1) is greater than 0.1 . The distributions are characterized by heavy-tails: there are few very important input suppliers and few very important input demanders. The power law distribution of the centrality measures is usually interpreted in the network analysis literature as an evidence in favor of a robust-yet fragile feature (see Haldane et al., 2009). Robust-yet fragile feature means that a network is highly robust against the random removal of nodes, but it is fragile to the specific removal of the most highly connected nodes. If an important node on the network suffers an attack, the whole network is affected. In the economic literature, an

\footnotetext{
${ }^{6}$ As noted in Clauset et al. (2009), only few empirical distribution follow a power-law for all values of $x$. More often, the power law distribution applies only for values of $x$ greater than a given threshold $x_{m i n}$, implying that the tail of the distribution follows a power-law.
} 
input-output network with heavy tails implies that idiosyncratic shocks can influence the whole economic system. The important nodes have a crucial role in shaping the aggregate behavior.

In this paper we claim that the topology of a network is very important to understand the properties of the system, but also that there is the need for an explicit model describing the economic role of nodes and links to fully understand the influence of each node. In order to formalize a precise economic interpretation of the network we describe a theoretical model in which firms' maximizing behavior determines quantities and prices. The WIOT network will be used to calibrate the technological parameters of the model, so that the flows of goods produced by the model are exactly the same as the observed ones.

Using the model, we can shock the total factor productivity of each sector. The influence of the shock on the aggregate system measures the model-based importance of the sector. The comparison of the model-based importance with the centrality measures described in the previous sections will show the importance of the theoretical model for the interpretation of the network topology.

\section{The model}

In this section we describe a model in the spirit of Long Jr and Plosser (1983) and Acemoglu et al. (2012) to give a theoretical structure to the input-output network. The parameters of the production functions and households' preferences will to be calibrated to reproduce the observed nominal trade flows in the WIOT 2011.

We assume that the economy is composed by $N$ sectors, and that each sector is represented by a representative firm technology:

$$
x_{i}=z_{i} l_{i}^{\alpha_{i}} \prod_{j=1}^{N} x_{i, j}^{\left(1-\alpha_{i}\right) w_{i j}}
$$

$x_{i}$ is the output of the $i-t h$ firm/sector, $z_{i}$ is total factor productivity, $l_{i}$ is labor employed by firm $i, \alpha_{i}$ is the share of labor in sector $i, 0<\alpha_{i}<1, x_{i j}$ is the amount of good $j$ used in the production of $i,\left(1-\alpha_{i}\right) w_{i, j}$ is the share of input $j$ in the production of $i$. We assume that $w_{i j} \geq 0$ and constant return to scale, i.e. $\sum_{j} w_{i j}=1$. The values of $w_{i j}$ determine the technological network in the economic system. If $w_{i j}>0$ there is a link with weight $w_{i j}$ between firm $i$ and firm $j$, i.e. firm $i$ is using good $j$ to produce and there is a flow of goods from firm 
$j$ to firm $i$. If $w_{i j}=0$ no link is present since firm $i$ is not using good $j$ for production. The matrix $\mathcal{W}$ containing all the elements $w_{i j}$ is an adjacency matrix representing a directed and weighted network, where the nodes are the firms and the links represent the technological links between the production functions.

The household sector is represented by one representative individual which supplies inelastically $\bar{l}$ unit of labor and maximizes the following utility function

$$
\mathcal{U}=\prod_{i=1}^{N} x_{i}^{c_{i}}
$$

where $x_{i}$ is the quantity of good $i$ consumed by the household, $c_{i}$ is a preference parameter for good $i, c_{i} \geq 0$ and $\sum_{i} c_{i}=1$, under the budget constraint:

$$
\sum_{i} x_{i} p_{i} \leq \bar{l} \eta
$$

where $\bar{l}$ is the inelastic labor supply and $\eta$ is the unitary nominal salary, $p_{i}$ is the price of good $i$. The resulting demand function for $\operatorname{good} i$ is

$$
x_{i}^{d}=\frac{\bar{l} \eta c_{i}}{p_{i}}
$$

\subsection{Equilibrium}

The system is in equilibrium when all markets are cleared, households maximize their utility and firms maximize their profits. We assume that in each sector there is an infinite number of perfectly competitive firms. This implies that the price set in each sector equals the marginal cost. We assume that the labor supply is inelastically equal to $\bar{l}=1$. Optimal demand for inputs derived from the maximization of profits are the following:

$$
\begin{gathered}
x_{i j}=\frac{\left(1-\alpha_{i}\right) w_{i j} p_{i} x_{i}}{p_{j}} \\
l_{i}=\frac{\alpha_{i} p_{i} x_{i}}{\eta}
\end{gathered}
$$


Substituting equation (5) and equation (6) in the production function and taking the logs we obtain:

$$
\begin{aligned}
\alpha_{i} \log \eta & =\log \left(z_{i}\right)+\alpha_{i} \log \alpha_{i}+\alpha_{i} \log p_{i}+\left(1-\alpha_{i}\right) \log p_{i}+ \\
& +\left(1-\alpha_{i}\right) \log \left(1-\alpha_{i}\right)-\left(1-\alpha_{i}\right) \sum_{j} w_{i j} \log p_{j}+\left(1-\alpha_{i}\right) \sum_{j} w_{i, j} \log w_{i j}
\end{aligned}
$$

setting $A_{i} \equiv \alpha_{i} \log \alpha_{i}+\left(1-\alpha_{i}\right) \log \left(1-\alpha_{i}\right)+\left(1-\alpha_{i}\right) \sum_{j} w_{i, j} \log w_{i j}$ we get the optimal price of firm $i$

$$
\log p_{i}=-\log \left(z_{i}\right)+\left(1-\alpha_{i}\right) \sum_{j} w_{i j} \log p_{j}-A_{i}-\alpha_{i} \log \eta
$$

Letting $\hat{\mathcal{W}}=\left((1-\alpha) \mathbf{1}^{\prime}\right) \circ \mathcal{W}$, where $\circ$ is the Hadamard product operator, the optimal price in vectorial form is:

$$
\log p=-\log (z)+\hat{\mathcal{W}} \log p-A-\alpha \log (\eta)
$$

where symbols without subscript $i$ denote $N \times 1$ column vectors, implying

$$
\log p=(I-\hat{\mathcal{W}})^{-1}(-\log (z)-A-\alpha \log \eta)
$$

Using the market clearing conditions we compute the equilibrium quantities. In particular the market clearing condition for good $i$ implies that total production equals the demand for consumption goods and inputs:

$$
x_{i}=\frac{\eta c_{i}}{p_{i}}+\frac{(1-\alpha)}{p_{i}} \sum_{j} w_{j i} p_{j} x_{j}
$$

where we have used $\bar{l}=1$. Multiplying both sides by $p_{i}$

$$
p_{i} x_{i}=\eta c_{i}+(1-\alpha) \sum_{j} w_{j i} p_{j} x_{j}
$$

and

$$
s_{i}=\eta c_{i}+(1-\alpha) \sum_{j} w_{j i} s_{j}
$$

where $s_{i} \equiv p_{i} x_{i}$ is the equilibrium value of sales for sector $i$. Thus, the vector of equilibrium sales is the following:

$$
s=\eta c^{\prime}[I-\hat{\mathcal{W}}]^{-1}
$$


Using the equilibrium vector of prices, the equilibrium production is $x_{i}=s_{i} / p_{i} \forall i$.

We calibrate the model setting $N$ equal to the number of sectors in the WIOT 2011 matrix and assuming that the entries of the WIOT 2011 matrix are equilibrium sales. The perfect competitive equilibrium implies that profits are zero in equilibrium and that the nominal GDP coincides with the nominal wage $\eta$. By using the WIOT 2011 matrix to set the technological parameters $\alpha_{i}, w_{i, j}$ and the preference parameter $c_{i}$, the model can reproduce the observed nominal input-output matrix. ${ }^{7}$ The calibrated model provides the equilibrium prices and the equilibrium quantities consistent with the observed input-output matrix. It is possible to determine the effect that a productivity shock to any sector has on prices and quantities of all the other sectors. The definition of shock depends on the model, and the effect of the shock is mediated by the assumptions about the behavior of the firms. How the nodes react to a shock is crucial to understand the behavior of the network. This is exactly the aim of this paper: to perform a network analysis using the observed network and characterizing the relations between sectors using an economic model.

\section{On the dynamics of price: the spread of a shock}

To determine the importance of sector $j$, we measure the effect that a shock to the productivity of sector $j$ has on the equilibrium price of all the other sectors in the network. To this aim we compute the derivative of the prices with respect to $z_{j}{ }^{8}$. Note that due to the assumption of perfect competition, a change in price is equivalent to a change in costs. By computing the derivative of prices, we are computing the cost effect of a shock to sector $j$. Recall the equation of the equilibrium log price vector:

$$
\log p=(I-\hat{\mathcal{W}})^{-1}(-\log (z)-A-\alpha \log \eta)
$$

and denote $\overline{\mathcal{W}} \equiv(I-\hat{\mathcal{W}})^{-1}$. The derivative of the logarithm of equilibrium prices with respect to $z_{j}$ is

$$
\frac{\partial \log p}{\partial z_{j}}=\overline{\mathcal{W}}_{\bullet, j} \frac{-1}{z_{j}}
$$

\footnotetext{
${ }^{7}$ For details see appendix B.

${ }^{8}$ Due to the Cobb-Douglas production function and utility function, the elasticity of demand of good $i$ with respect to the price $p_{i}$ is 1 . This implies that studying the effect of a shock on prices is equivalent to study the effect of a shock on quantities.
} 
where $\overline{\mathcal{W}}_{\bullet}, j$ is column $j$ of matrix $\overline{\mathcal{W}}$, which, since $p_{i}=e^{\log p_{i}}$, and $\frac{\partial p_{i}}{\partial z_{j}}=p_{i} \frac{\partial \log p_{i}}{\partial z_{j}}$, means that

$$
\frac{\partial p}{\partial z_{j}}=p \circ \overline{\mathcal{W}}_{\bullet, j} \frac{-1}{z_{j}}
$$

The effect of a shock to sector $j$ depends on the technological relationship between sector $j$ and all the other sectors in the network and on the optimal reaction of the firms to the change in the price of their inputs. The column vector $\overline{\mathcal{W}}_{\bullet, j}$ represents the direct and indirect weight of sector $j$ in the production of all the other goods in the economic system. With the following proposition we show that - in the assumed economic system - shocks are propagated only downstream.

Proposition 4.1 If the economic system is characterized by Cobb-Douglas production functions and utility functions, supply shocks propagate only downstream.

Proof To simplify the proof, we assume that the economic system is characterized by a directed and weighted input-output matrix, $A$, without circular links. This network topology allows the upstream-downstream relations to be clearly determined. Such a network is characterized by a lower-triangular adjacency matrix:

$$
A=\left(\begin{array}{cccc}
a_{1,1} & 0 & \cdots & 0 \\
a_{2,1} & a_{2,2} & \cdots & 0 \\
\vdots & \vdots & \ddots & \vdots \\
a_{N, 1} & a_{N, 2} & \cdots & a_{N, N}
\end{array}\right)
$$

where the element $a_{i, j}$ represents a link from node $j$ to node $i$. Note that node 1 supplies input to all nodes, node 2 supplies inputs to all nodes but node 1 , and so on. This implies that node 1 is upstream to all nodes and node 2 is upstream to all nodes but node 1 . For the properties of triangular matrices, the matrix $\bar{A}=(I-A)^{-1}$ is still a lower triangular matrix:

$$
\bar{A}=\left(\begin{array}{cccc}
\bar{a}_{1,1} & 0 & \cdots & 0 \\
\bar{a}_{2,1} & \bar{a}_{2,2} & \cdots & 0 \\
\vdots & \vdots & \ddots & \vdots \\
\bar{a}_{N, 1} & \bar{a}_{N, 2} & \cdots & \bar{a}_{N, N}
\end{array}\right)
$$

From eq.(16), we know that the derivative of the price of sector $i$ with respect to a productivity shock on sector $j$ is positive only if the element $\bar{a}_{i, j}$ is positive. This implies that the price 
derivative is positive only if sector $i$ is downstream with respect to sector $j$.

An example showing how shocks spread only downstream can also be found in Acemoglu et al. (2015). Computing eq. (16) for all sectors, we build the network of effects, where the element $(i, j)$ is $\frac{\partial \log p_{i}}{\partial z_{j}}$, i.e. the direct and indirect effect of a shock to sector $j$ on the price (and production cost) of sector $i$. In figure 2 we display the network of effects, where the sectors are arranged in a Country-Sector order ${ }^{9}$. Darker colors are associated with a greater (absolute) derivative. The figure reveals a clear community structure at country level shown by the darker block main diagonal. World production is organized so that shocks mainly propagate inside the sector of each country, while inter-country relationships are weaker. From figure 2 it is also possible to detect sectors with global influence. Darker columns correspond to sectors with a strong impact on world production. The economic model allows to transform the empirical

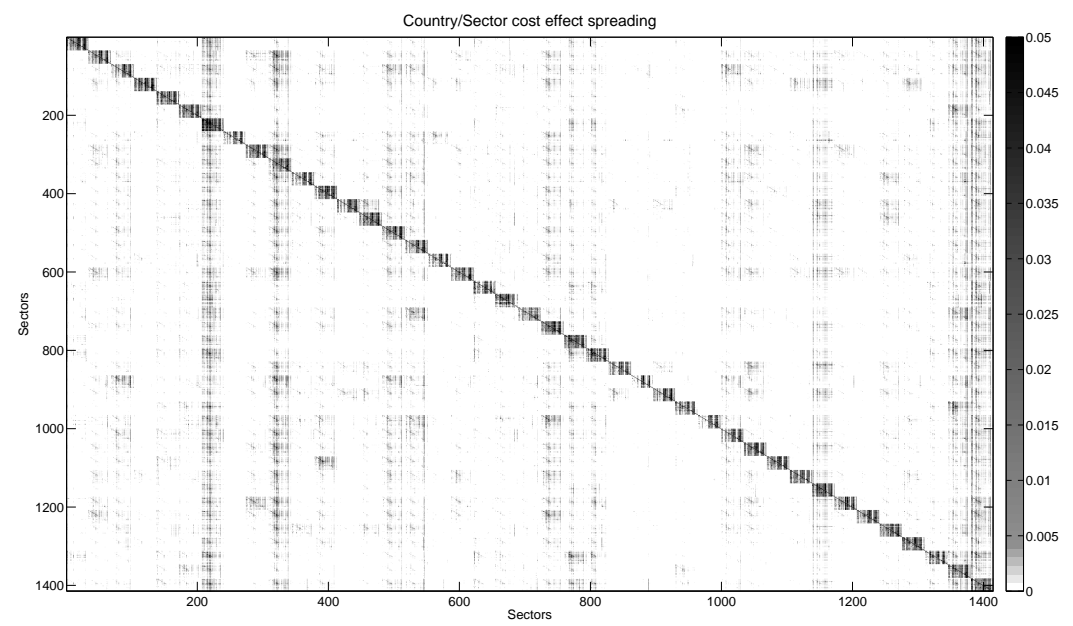

Figure 2: Derivatives matrix. The colors show the size of the derivative. The elements in column $j$ represent the derivative with respect to a shock to sector $j$. The sectors are sorted in a Country-Sector order.

matrix of nominal trade flows into a matrix of relations taking into account the technological links and the optimal behavior of the firms. The correlation between the elements in the WIOT matrix and the network of effects matrix is .24, meaning that the model is adding information on the relations between sectors ${ }^{10}$. The additional information can be explained in behavioral terms. A negative shock to sector $j$ implies an increase in the price of good $j$. Downstream firms react to the increase in cost of input $j$ by optimally substituting it with the other available inputs,

\footnotetext{
${ }^{9}$ For example countries $\mathrm{A}$ and $\mathrm{B}$ and sectors 1 and 2: the first column of the network of effects matrix would display the derivative with respect to sector A1, the second column the derivative with respect to sector A2, and so forth.

${ }^{10}$ The correlation between the elements of the WIOT network and the network of effects is computed stacking all columns of the matrices into two vectors and then calculating the correlation coefficients between these vectors. In this way we measure the similarity between the two matrices.
} 
dampening but not removing the impact of the shock. In order to have a deeper understanding
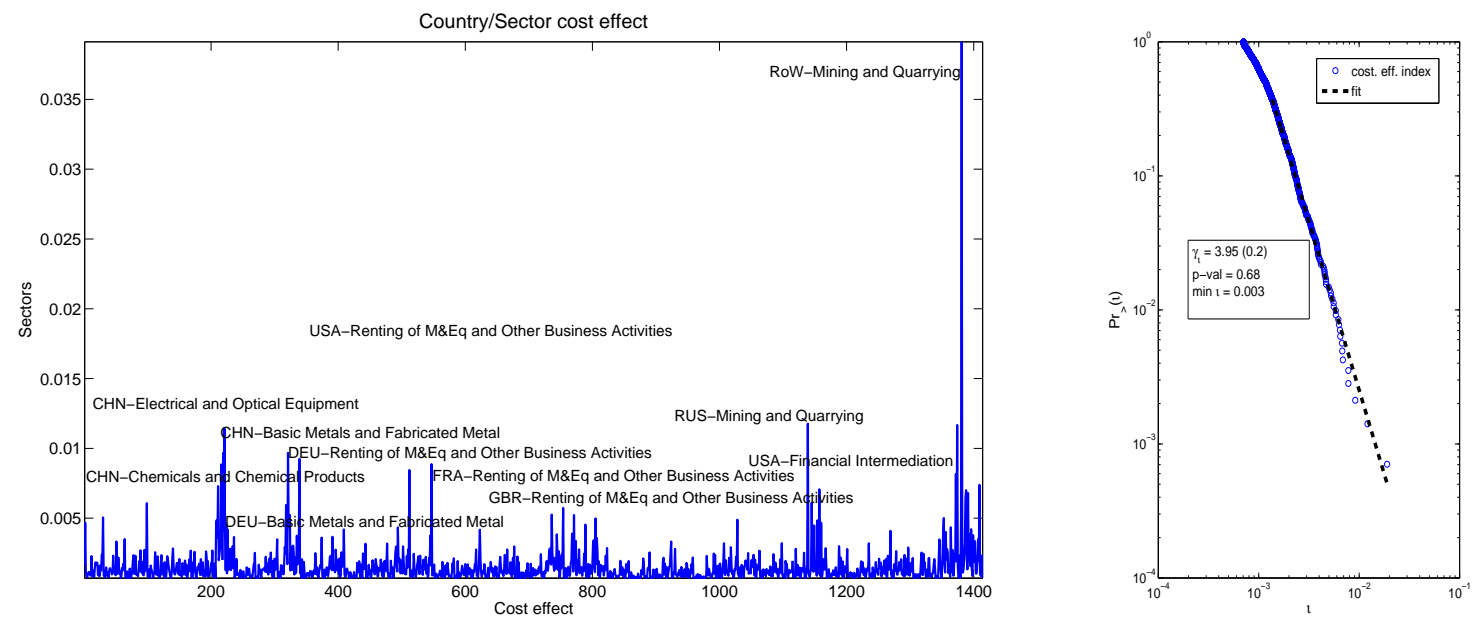

Figure 3: Cost effect index. Left: Cost effect index of each sector. The x-axis reports the 1414 sectors active in the WIOD dataset in 2011, the y-axis shows the cost effect index. Right: Log-log plot of the cumulative distribution function and maximum likelihood power-law fit for the cost effect index distribution.

of the global influence of each sector on the cost of the other sectors, we compute a cost effect index. The cost effect index of sector $j$ is the average change of production costs due to a shock to sector $j$ :

$$
\iota_{j}=\frac{1}{N} \sum_{i=1}^{N} \frac{\partial \log p_{i}}{\partial z_{j}}
$$

Note that $\sum_{i=1}^{N} \frac{\partial \log p_{i}}{\partial z_{j}}$ is equivalent to the out-strength of node $j$ in the network of effect and that $\iota_{j}$ is a measure of the average influence of node $j$. The cost effect index and its distribution are shown in figure 3. The cost effect index follows a power-law with exponent $\gamma_{\iota}=3.95(0.2)$ as reported in the right panel of figure 3 .

\subsection{Topological measures and cost effect index}

It is interesting to compare the cost effect index of each sector with its position in the inputoutput network. The cost effect index is influenced both by the position of sectors in the input-output network and by the assumptions made to build the model. When a shock hits sector $j$, the price of input $j$ increases. The downstream sector $i$ reacts instantaneously by substituting input $j$ with other inputs, thereby reducing the demand for input $x_{i j}$. Production costs (and the price) of sector $i$ increase, spreading the shock further down in the production chain. The assumptions in the model govern the behavior of the firms in the network and give a precise theoretical meaning to shock, and spreading. Due to the assumption of Cobb-Douglas production functions and Cobb-Douglas utility function, the shock spreads only to downstream 
firms, and not to upstream firms. The position of a sector as a demander does not matter to its cost effect index. Therefore, the topological measures of in-strength and input PageRank are useless to determine the systemic importance of a sector. The sectors that are systemically important in the global production network are those supplying inputs. In figure 4 we analyze the relationship between the cost effect index and the out-strength and output PageRank score of the sectors. The correlation between the centrality measures and the cost effect index is very

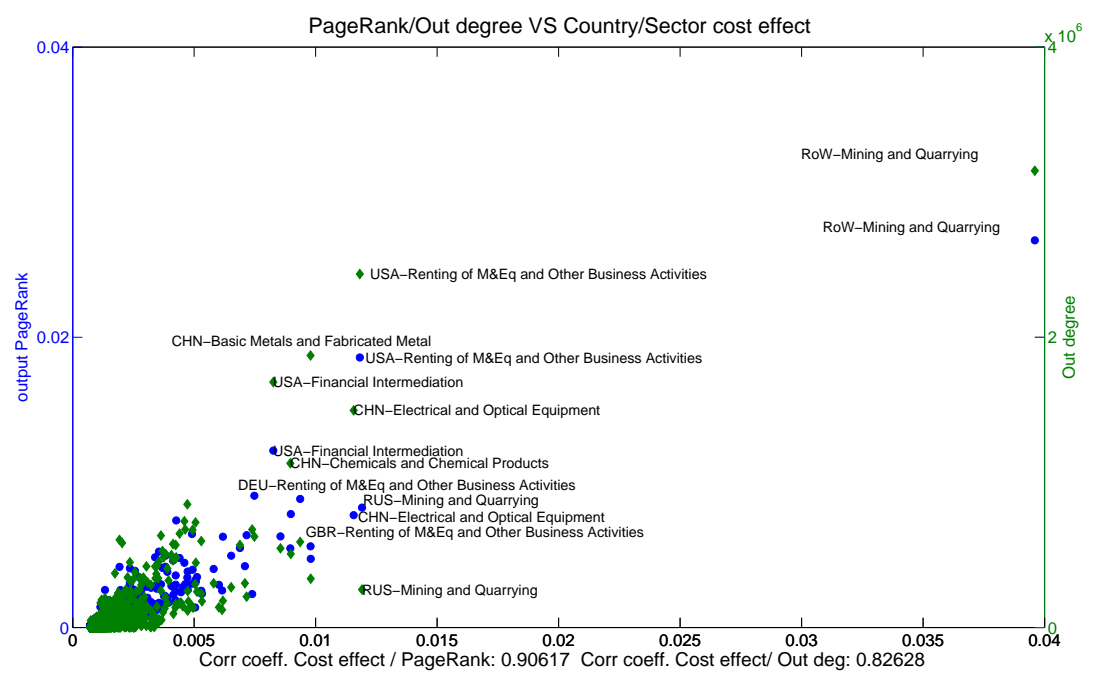

Figure 4: Cost effect index plotted against out-strength (green diamonds) and out-PageRank score (blue circles). Below the figure we report the correlation coefficients between the measures.

high, yet it is not 1, meaning that the model is adding information on the interaction between sectors. In particular the cost effect index correlates 0.90 with the output PageRank and 0.82 with the out-strength. The global centrality measure, i.e. the output PageRank, better explains the cost effect index. The interpretation of the result is related to the nature of the shock and to the reaction of the sectors to the shock. The initial shock is spread through the network as a cascade from upstream to downstream sectors. Therefore, the cost effect index is high if the sector hit by the shock has many customers, and/or if the sector hit by the shock is linked with other sectors having many customers. The output PageRank score takes into account both the direct effect on the neighbors of $j$, and the indirect effect depending on the characteristics of the neighbors' neighbors of sector $j$.

Mining and Quarrying sector from the Rest of the World has a huge effect on global production costs because it provides most of the raw materials to world manufacturers. However, it is striking that together with providers of raw materials, the most important sectors are Financial Intermediation and Renting of Machines and Equipment sectors. This result highlights the 
strategic position of business services in the global production chain and clearly shows that the health of the financial system is crucial for the well-being of the global economic system.

\section{The effect on the real GDP}

In this section we develop an index measuring the effect of a shock to a given sector on global real GDP. Since the utility function is Cobb-Douglas, we can define a price index as follows:

$$
\mathcal{P}=\prod_{i=1}^{N}\left(\frac{p_{i}}{c_{i}}\right)^{c_{i}}
$$

where $c_{i}$ is set equal to the share of nominal GDP consumed in each sector in the WIOT database. In a perfect competitive equilibrium, where total labour $\bar{l}=1$, the nominal GDP coincides with the nominal wage $\eta$. Thus, the real GDP is

$$
y=\frac{\eta}{\mathcal{P}}
$$

The derivative of the real GDP with respect to the total factor productivity of sector $j, z_{j}$, is:

$$
\frac{d y}{d z_{j}}=\frac{\partial y}{\partial \mathcal{P}} \sum_{i} \frac{\partial \mathcal{P}}{\partial p_{i}} \frac{\partial p_{i}}{\partial z_{j}}
$$

which turns out to be

$$
\frac{d y}{d z_{j}}=-\frac{\eta}{\mathcal{P}} \sum_{i} c_{i} \frac{\partial \log p_{i}}{\partial z_{j}}
$$

The effect on the real GDP of a shock to sector $j$ is a weighted sum of the price derivatives with respect to $z_{j}$. The weights are represented by preference parameters in the utility function. We compute the GDP effect index of sector $j$ by normalizing $\frac{d y}{d z_{j}}$ by the number of sectors:

$$
\varkappa_{j}=\frac{1}{N} \frac{d y}{d z_{j}}
$$

The effect on the real GDP reflects also the effect of the shock on the utility of the representative household. We can therefore interpret the GDP effect index as a welfare effect index. The GDP effect index for each sector is shown in the left panel of figure 5, and its distribution is shown in the right panel of figure 5. The GDP effect is distributed as a power-law with exponent $\gamma_{\varkappa}=2.88(0.05)$. Figure 6 shows the correlation between the GDP effect index and the output 

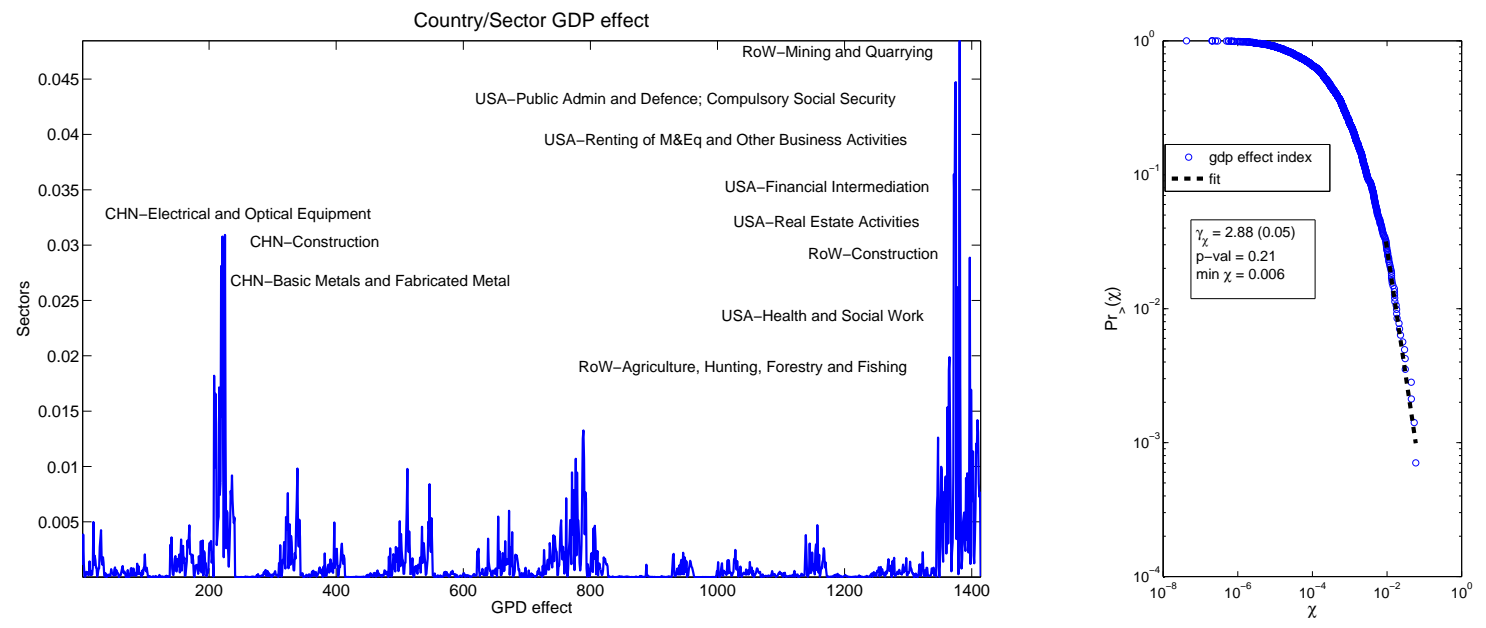

Figure 5: GDP effect index. Left: the x-axis reports the sectors, the y-axis reports the GDP effect index. Right: Log-log plot of cumulative distribution function and maximum likelihood power-law fit for the GDP effect index distribution.

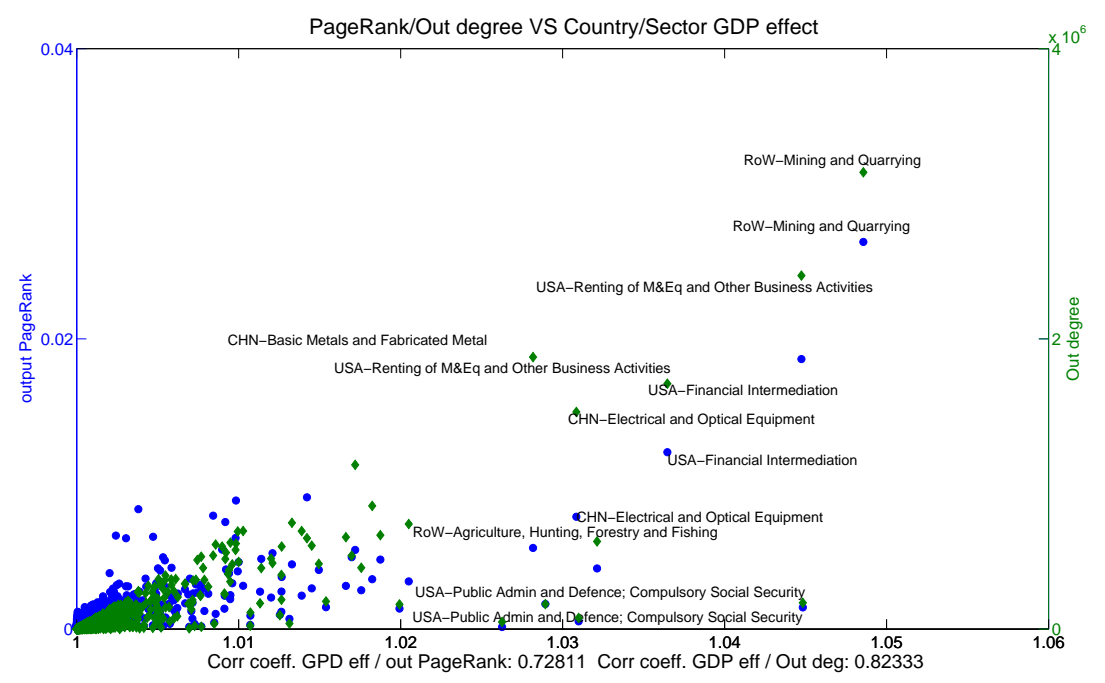

Figure 6: GDP effect index plotted against out-strength (green diamonds) and out-PageRank score (blue circles). Below the figure we report the correlation coefficients between the measures. 
PageRank score (blue circles) and the out-strength (green diamonds) for each sector. The GDP effect index and the output PageRank score have a correlation of 0.72 , and the GDP effect index and the out-strength have a correlation of 0.82 . The result is reversed with respect to the cost effect index: the local centrality measure (out-strength) explains better the influence of a sector on the world real GDP with respect to the global centrality measure. The interpretation is the following. The spreading of the shock through the network in terms of real GDP is dampened by the high probability of encountering low values of the utility parameter $c_{i}$, implied by the power-law distribution of the utility parameter (see right panel of figure 1). Therefore, the higher the out-strength, the higher the probability that the shock will directly hit a sector with high $c_{i}$ value. In this regard, the proximity to the consumption sector is important to determine the GDP effect index. The USA-Public Administration has few links within the input-output network, however a shock to this sector would have a great influence on the real GDP. Similarly, the Agricultural sector in the rest of the world (ROW) has a huge impact on the utility of households, despite having relatively low importance in the input-output network.

\section{The evolution of the global input-output network}

One of the most important properties of a network is its resilience to shocks. To analyze the evolution of the fragility of the global input-output network we calibrate the model using the WIOT data from 1995 to 2011. In order to have a better comparability of the results over time we keep the nominal GDP equal to 1. This is equivalent to normalizing the output of the model, and all trading flows, by the nominal wage. ${ }^{11}$

We define a fragility index as the expected cost effect of a random shock, i.e. a shock hitting a random node:

$$
I=\frac{1}{N} \sum_{j} \iota_{j}
$$

where $\iota_{j}$ is defined in eq.(18) and represents the effect of a shock to sector $j$ on the global production cost. The fragility index can also be written as the expected cost effect index $I=E\left(\iota_{j}\right)$. Similarly we define a fragility index using the GPD effect index defined in eq.(22):

$$
K=\frac{1}{N} \sum_{j} \varkappa_{j}
$$

\footnotetext{
${ }^{11}$ Since we assume perfect competition and inelastic labor supply equal to 1, the world nominal GDP is equivalent to the aggregate wage bill.
} 
In figure 7 we show the evolution of the fragility indexes $I$ and $K$ from 1995 to 2011 . The evidence suggests that the fragility of the world input-output network has increased over time. The fragility index is computed as the average cost effect index in each year ${ }^{12}$; therefore an increase in the fragility index is caused by an increase in the average price derivative. The price derivative is determined in eq. (16) and depends on the columns of the matrix $\overline{\mathcal{W}}_{\bullet}, j$ for $\forall j$. Given the construction of the of $\overline{\mathcal{W}}_{\bullet}, j$, we know that an increase can be caused either by a change in the network or by a generalized decrease in $\alpha_{i}$. The increase of the fragility of
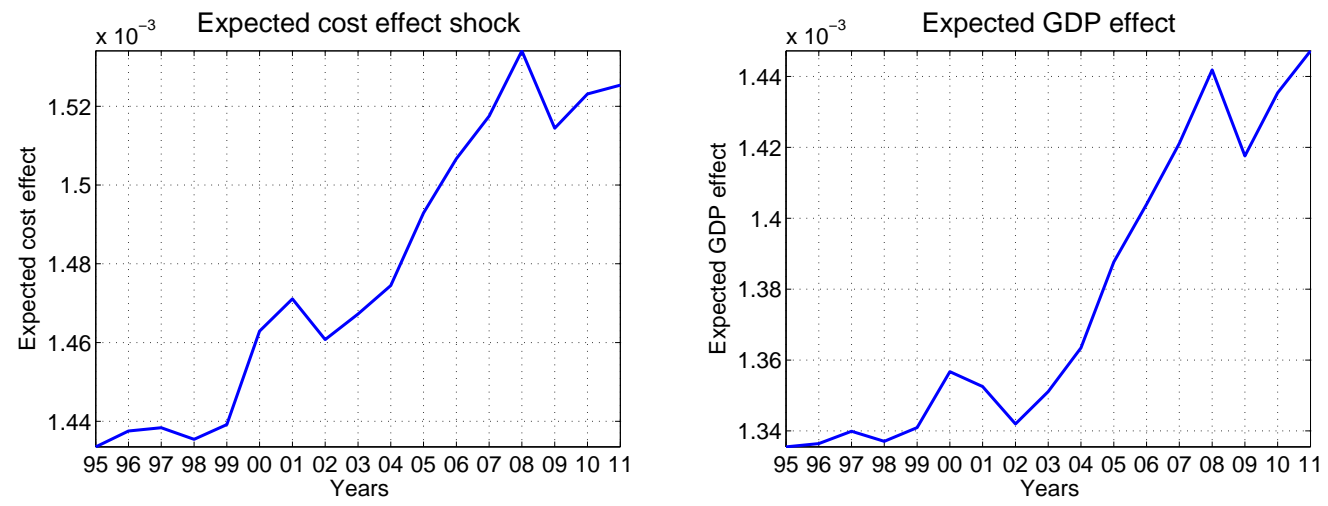

Figure 7: Left: The evolution of the expected cost effect, eq. (24). Right: The evolution of the expected GDP effect, eq. (25).
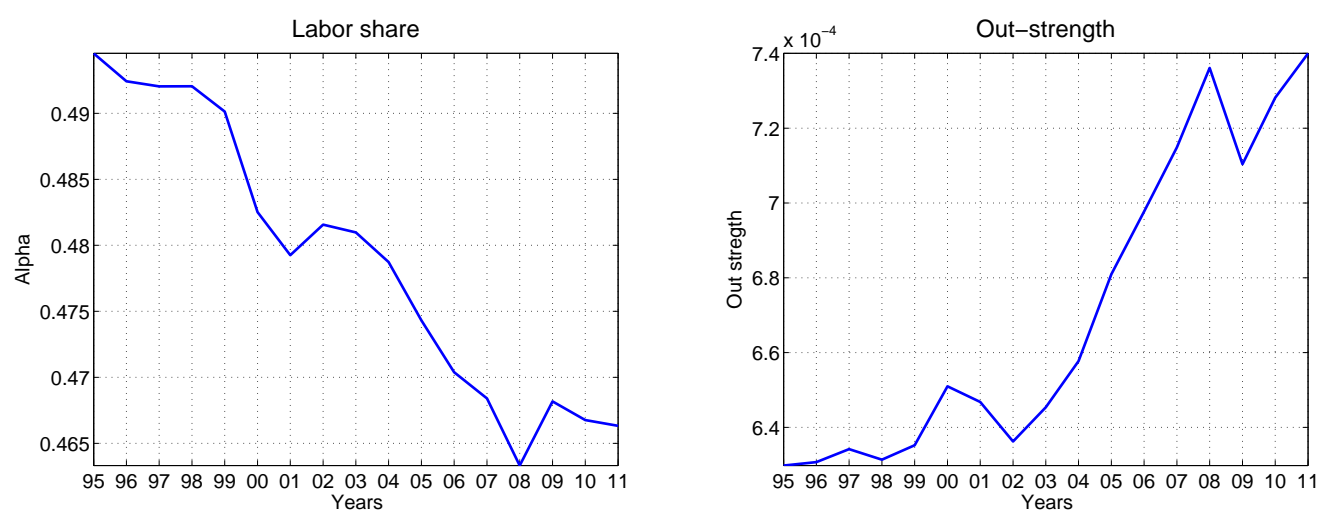

Figure 8: Left: The evolution of the average labor share $\alpha_{i}$. Right: Sum of the total out-strength relative to total GDP. The nominal value of inputs relative to nominal value added increased from 1995 to 2011.

the production network is thus caused by a change in the production process. The right panel of figure 8 shows the sum of the out-strength in each year, which is equal to the total value of inputs employed in the production. Since we are normalizing all trading flows by the GDP, by computing the sum of the out-strength of the input-output network we are determining the total amount of inputs necessary to produce one unit of nominal GPD ${ }^{13}$. The relative value

\footnotetext{
${ }^{12} \mathrm{~A}$ shock randomly hitting the economic system would have an expected cost effect equal to the average cost effect index

${ }^{13}$ Recall that we set the nominal wage to 1 , imposing the wage as numéraire.
} 
of inputs with respect to nominal wage has increased. The increase in the relative value of intermediate inputs is due to a change in the production technology, as production becomes more input intensive. In this regard, the left panel of figure 8 shows the average value of $\alpha_{i}$ in each year. As the average value of $\alpha_{i}$ decreases, the share of labor is reduced and consequently the share of intermediate inputs is increased. The increased importance of intermediate inputs in production implies a greater influence of the input-output network on the production cost. As the relative importance of inputs increases, the effect of a shock on the input-output network increases.

\subsection{New sectors on the block}

The evolution of the global production network is characterized by the rise of new central sectors. The increase in the cost effect index has not been uniform across sectors. Some sectors have increased their importance in global production, others have reduced their importance. To analyze how the role of sectors in the production network has changed over time, we compute in each period the share of the cost effect index of each sector, i.e. $\gamma_{j, t}=\iota_{j, t} / \sum_{i} \iota_{i, t}$. The change in $\gamma_{j, t}, \Delta \gamma_{j, t}=\gamma_{j, t}-\gamma_{j, t-1}$, denotes a change in the relative importance of sector $j$ from period $t-1$ to period $t$. Computing the sum $\Delta \gamma_{j, t}$ from 1996 to 2011 for each sector, we determine the change in the sectors' relative importance. The results are shown in figure 9. Some sectors that were peripheral in 1995, became very influential in 2011 . The most

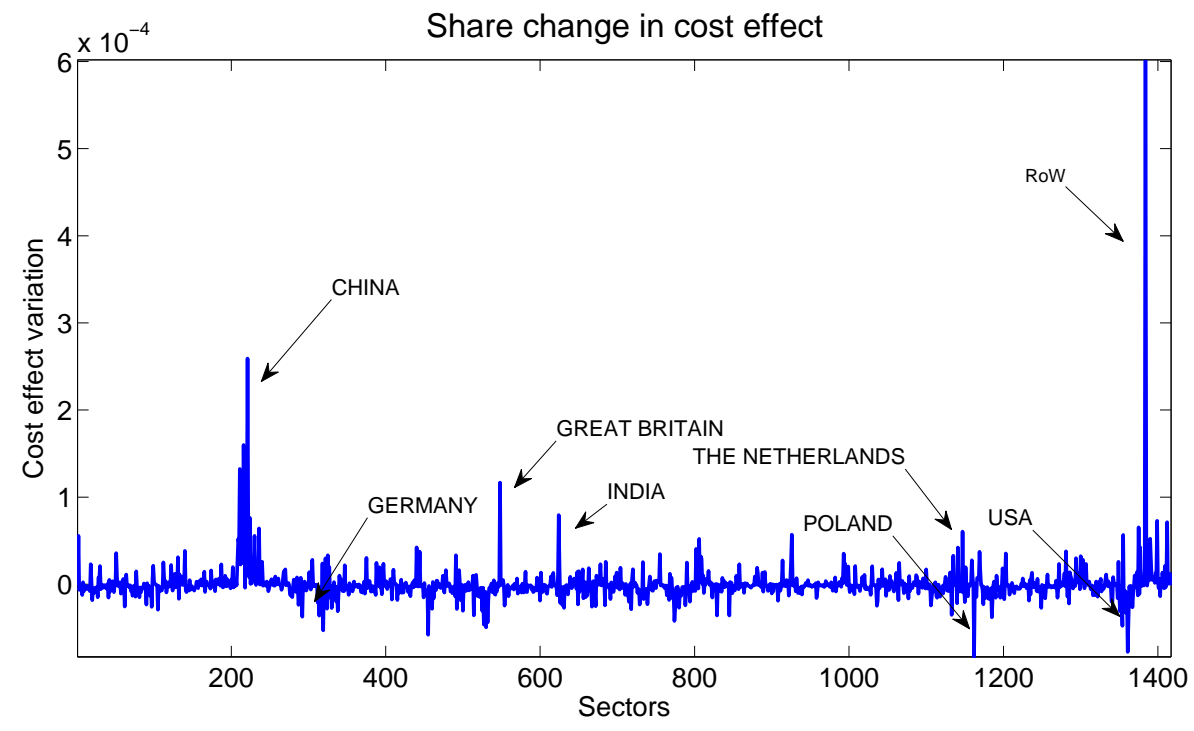

Figure 9: Total change in sectors' relative importance from 1995 to 2011.

spectacular change occurs in the Chinese production system, which gains an unprecedented 
centrality in global production. On the contrary, US sectors have lost influence. To show the Chinese centralization dynamics, we rank all sectors (from the highest to the lowest) with respect to the cost effect index and the GDP effect index in each year, and we analyze the evolution of the ranking of the 10 most central sectors in 2011. The left panel of figure 10 shows that some of the most central sectors in 2011 were peripheral sectors in 1995 . This is particularly true for Chinese Electrical and Optical Equipment, growing from the 137th position in 1995 to the 4th in 2011 (and from the 133rd to the 7th in the GDP effect index ranking, not shown). Both for the cost centrality measure and the GDP centrality measure, the Chinese production system has increased its importance, becoming the most influential economic system on a global scale. China was the most represented country in the top 10 cost effect index ranking in 2011 and the second most represented country in the top 10 GDP effect index ranking. Noticeably, the only new OECD country sector in the top 10 of the cost effect index, is the British Renting of M\&Eq and Other Business Activities, showing that the evolution of the network has been toward a global role diversification. The leading Chinese sectors are intermediate manufactured goods, while the western leading sectors are financial intermediation and business services, denoting the different role in the production network. The globalization of production has fostered an international division of production. To analyze
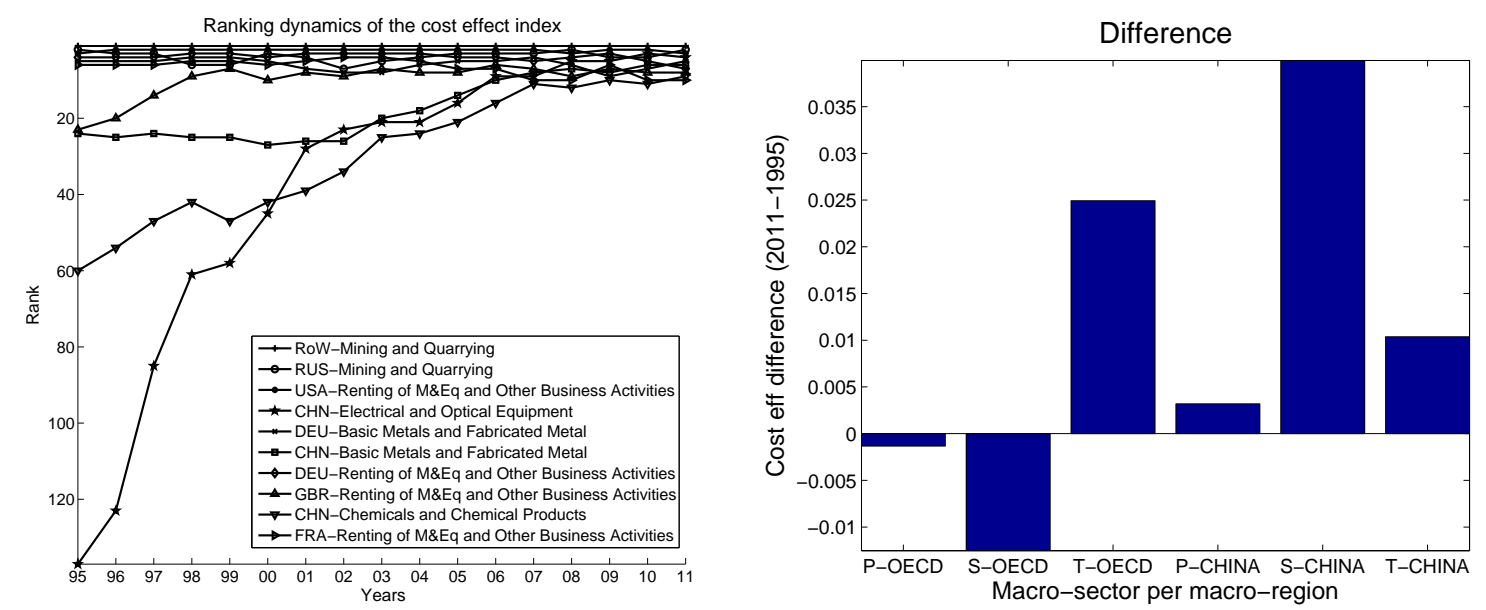

Figure 10: Left: Time evolution of the ranking of the 10 sectors with the highest cost effect index. The x-axis displays the years, the y-axis the position in the ranking. Some of the most influential sectors in 2011, were non-influential sectors in 1995. Right: The change in cost effect index from 1995 to 2011 for primary (P), secondary (S) and tertiary (T) macro sectors for OECD and China

this general pattern in world production organization, we aggregated all sectors in primary, 
secondary and tertiary sectors ${ }^{14}$ and computed the aggregated change in cost effect index in China and OCSE. The results are shown in the right panel of figure 10. From 1995 to 2011 world production organization has evolved. The importance of the secondary sector in OECD countries has declined, while the tertiary sector has increased its global influence. Manufacturing activities have been relocated in China, as shown by the centralization of the Chinese secondary sector. This result suggests that globalization is reorganizing production on a global scale. The increasing efficiency of transportation systems and information networks allows supply chains to spread all over the world.

\section{Conclusions}

In this paper we have analyzed the world input-output network through the lenses of a simple calibrated economic model. By defining the nodes as firms and the links as optimal flows of inputs, we can study the network with a proper theoretical framework. The empirical network can describe the topology of the interactions between economic agents, but the effect of this interaction has to be analyzed with the guidance of a formal model. By changing the economic model, we could change the way in which the shocks spread into the system and therefore the interpretation of centrality in the network. When a shock hits an economic system, it is not hitting an inert body. Economic agents react to the shock, and how they react to the shock determines the propagation mechanism. In the paper we show that the definition of an underlying model provides a meaningful economic interpretation to the shock and to the propagation mechanism. In particular, we show that when the economic system is characterized by Cobb-Douglas production functions, supply shocks can only propagate downstream.

We firstly analyzed the flow of trade network in 2011, finding that it is characterized by heavy tail distributions both of nodes out-strength and nodes in-strength. This implies that the input-output network is highly asymmetrical as regards both input suppliers and input users. Moreover, we also found that the distribution of consumption shares has heavy tails. From the macroeconomic literature (Horvath, 1998, 2000; Acemoglu et al., 2012), we know that the asymmetry in the input-output network is exactly the condition that renders the inputoutput interaction important in determining the aggregate outcome of the economic system. In particular, only few huge sectors are going to have an impact on the aggregate system. To

\footnotetext{
${ }^{14}$ Following the ISIC Rev.3 classification we included in the primary sector all sectors classified A,B and C, in the secondary sector all sectors classified from D to $G$, and in the tertiary all the remaining sectors.
} 
understand which are the most influential sectors in the production network, we defined two indexes: the cost effect index and the GDP effect index. The cost effect index measures the influence of a sector on global production costs. The cost effect index is clearly dependent on the production function and on the optimizing behavior of the firms. Firms optimally substitute their inputs to minimize their costs. When a shock hits the system all nodes will react to the change in production costs. The intensity of the reaction depends on the position of the sector in the supply chain. The cost effect index takes into account the direct and indirect effect of a shock, and provides complete and model-based information on the influence of a sector on the global production process. The GDP effect index measures the influence of a sector on global real GDP. The definition of real GDP depends on the definition of price index, and in turn on the definition of utility function. We show that the importance of a sector in influencing the real GDP crucially depends on its own share in consumption and on the out-strength. The propagation process is less important in explaining the GDP effect index.

The second research question analyzed in this paper regards the evolution of the inputoutput network. We found that the network increased its fragility from 1995 to 2011. This can be explained by a change in the production process, and in particular it is caused by an increase in the relative weight of intermediate inputs in the production. The higher the relative value of the intermediate inputs in production with respect to labor input, the higher the effect of a shock to the intermediate inputs production.

The evolution of the global production network has been characterized also by the rise of new central sectors. The most spectacular change occurred in the Chinese production system, which gained an unprecedented centrality in global production. China is the most represented country in the top 10 cost effect index ranking in 2011 and the second most represented country in the top 10 GDP effect index ranking. It is also very interesting to note how the leading Chinese sectors are intermediate manufactured goods, while the western leading sector are financial intermediation and business services, denoting the different role in the production network. This change in global production organization has also been confirmed by the change in the aggregate global influence of the secondary sectors of OECD countries and China, as well as the change in the tertiary sectors. While the OECD countries manufacturing has decreased its importance, OECD countries services, and in particular financial and business services have increased their global influence.

This model-based network analysis improves the understanding of the world input-output 
network, with respect to a purely empirical network analysis. Future research should test different models to understand the implications of different behavioral assumption and technologies on the sector centrality measures.

\section{Acknowledgements}

The research leading to these results has received funding from the European Union, Seventh Framework Programme FP7/2007-2013 Socio-economic Sciences and Humanities under Grant Agreement No. 612796 MACFINROBODS and under Grant Agreement No. 320278 RASTANEWS. 


\section{Appendix A PageRank algorithm}

The PageRank computes the probability that a random walker will land on a given node. Suppose that each unit of input in the system moves according to a Markov process defined by an $N \times N$ transition probability matrix $\mathbf{p}=[\mathbf{p}]_{i j}$. Under a regularity condition (ergodicity of $\mathbf{p})$, there exists a real, positive vector $\pi_{i n}=\left[\pi_{i n}\right]_{i}, i=1, \ldots, N$ such that $\pi_{i n}=\mathbf{p} \pi_{i n}$ and $\sum \pi_{i n}(i)=1$. This is the PageRank vector. When $\mathbf{p}$ is not ergodic, one typically assumes that with some small probability a unity of input moves from any $i$ to any $j$, so $\pi_{i n}$ exists. The input PageRank is formally defined as

$$
\pi_{i n}=\varepsilon\left(\mathbf{W} \mathbf{\Phi}+\mathbf{f d}_{\text {out }}^{\prime}\right) \pi_{i n}+(1-\varepsilon) \mathbf{f}
$$

The parameter $\varepsilon \in(0,1)$ is a damping parameter that determines the relative importance of the matrix $\left(\mathbf{W} \mathbf{\Phi}+\mathbf{f d}_{\text {out }}^{\prime}\right)$ and the teleportation distribution $\mathbf{f} . \mathbf{W}$ is the weighted adjacency matrix and $\boldsymbol{\Phi}$ is a diagonal matrix with elements $\boldsymbol{\Phi}_{i i}=\min \left(\frac{1}{k_{\text {out }, i}}, 1\right)$. The second component is $\mathbf{f d}_{\text {out }}^{\prime}$ where $\mathbf{d}_{\text {out }}$ is a column vector with elements $\mathbf{d}_{\text {out }, i}=1$ if $k_{\text {out }, i}=0$ and otherwise 0. The vector $\mathbf{d}_{\text {out }}$ identifies those individuals that have no outgoing links and avoids that the random walker 'gets stuck' on a dead-end node. Furthermore, not all nodes in the network are necessarily directly connected to one another. Therefore, the PageRank is adjusted again so that with probability $(1-\varepsilon)$ the walker is allowed to jump to any other node in the network according to $\mathbf{f}$. This is the reason why the vector $\mathbf{f}$ is called the teleportation distribution.

Since the matrix $\mathbf{W}$ is asymmetric; as with the case of the in- and out-degree, it is possible to derive two measures of centrality by looking at the transpose of $\mathbf{W}$. The output PageRank is formally defined as follows:

$$
\pi_{\text {out }}=\varepsilon\left(\mathbf{W}^{\prime} \mathbf{\Psi}+\mathbf{f d}_{\text {in }}^{\prime}\right) \pi_{\text {out }}+(1-\varepsilon) \mathbf{f}
$$

now $\mathbf{\Psi}_{i i}=\min \left(\frac{1}{k_{i n, i}}, 1\right)$ and $\mathbf{d}_{i n, i}=1$ if $k_{i n, i}=0$ and 0 otherwise. 


\section{Appendix B Data and calibration}

\section{B.1 WIOT}

The World Input-Output Table (WIOT) is a database providing the input-output tables for 40 countries $(27 \mathrm{EU}$ countries and 13 other major countries in the world and a model for the rest-ofthe-world) and 35 industries and a set of final consumption sectors for each country, covering the period from 1995 to 2011 (Timmer et al., 2012). We aggregate the final consumption sectors in one global sector and transpose the original WIOT data to make the input-output table consistent with the notation used in the model described in section 3. WIOT provides data on the flow of inputs between sectors, the value of consumption and the value added for each sector. Column $i$ provides the nominal flow of goods from sector $i$ to the other sectors and the flow of goods from sector $i$ to the final consumption sector. The sum of column $i$ provides the total use of good $i$. Row $i$ provides the inputs flowing into sector $i$ and the value added produced by sector $i$. The sum of nominal intermediate inputs used by sector $i$ plus the value added in sector $i$, i.e. the sum over row $i$, gives the total value of production of sector $i$. The sum of the value added column gives the nominal GDP. A general representation of the input-output data is shown in table 1 .

\begin{tabular}{|c|c|c|c|c|c|c|}
\hline sectors & sector 1 & sector 2 & sector $n$ & $\begin{array}{l}\text { value } \\
\text { input }\end{array}$ & $\begin{array}{l}\text { value } \\
\text { added }\end{array}$ & $\begin{array}{l}\text { value } \\
\text { production }\end{array}$ \\
\hline $\begin{array}{l}\text { sector } 1 \\
\text { sector } 2 \\
\text { sector } n\end{array}$ & $\begin{array}{l}W_{1,1} \\
W_{2,1} \\
W_{n, 1}\end{array}$ & $W_{1,2}$ & $W_{1, n}$ & $\sum_{j=1}^{n} W_{1, j}$ & $v a_{1}$ & $Y_{1}$ \\
\hline value intermediate & $\sum_{i=1}^{n} W_{i, 1}$ & & & & & \\
\hline final consumption & $D_{1}$ & & & & & \\
\hline value use & $D_{1}+\sum_{i=1}^{n} W_{i, 1}$ & & & & & \\
\hline
\end{tabular}

Table 1: Input-output table

\section{B.2 Calibration}

The model described in section 3 assumes market clearing and perfectly competitive markets. The former assumption implies that total nominal production is equal to total nominal demand. The latter assumption implies zero profits, and that value added in each sector is equal to the wage bill. Assuming that total labor is 1 , the nominal GDP is equal to the nominal salary. We 
assume a Cobb-Douglas production function:

$$
x_{i}=z_{i} l_{i}^{\alpha_{i}} \prod_{j=1}^{N} x_{i, j}^{\left(1-\alpha_{i}\right) w_{i j}}
$$

where $\alpha_{i}$ is the optimal share of labor, and $\left(1-\alpha_{i}\right) w_{i, j}$ is the optimal share of input $j$. The model is therefore reproducing the WIOT data by setting $\alpha_{i}$ equal to the observed share of labor:

$$
\alpha_{i}=\frac{v a_{i}}{Y_{i}} \forall i
$$

where $v a_{i}$ is the value added in sector $i$, and $Y_{i}$ is the total nominal production of sector $i$. Noting that $\left(1-\alpha_{i}\right) w_{i j}$ is the share of input $j$, we can set

$$
w_{i, j}=\frac{W_{i, j}}{\sum_{j} W_{i, j}} \forall i
$$

the weight of sector $j$ in the production of good $i$ is determined as the share of input $j$ on the total use of intermediate inputs by sector $i$. Assuming an inelastic total supply of labor equal to 1 implies that total nominal GDP is equal to the nominal wage. Market clearing implies that nominal wage is equal to nominal consumption. In the data this assumption does not hold due to saving. However, we force market clearing and we set the nominal wage, $\eta$, equal to the observed sum of nominal consumption:

$$
\eta=\sum_{i} C_{i}
$$

where $C_{i}$ is the nominal consumption sector $i$. Households have a Cob-Douglas utility function:

$$
\mathcal{U}=\prod_{j=1}^{N} x_{i}^{c_{i}}
$$

Given the Cobb-Douglas consumption function, the share of budget spent on good $i$ is $c_{i}$. We set the utility parameter equal to:

$$
c_{i}=\frac{C_{i}}{\eta}
$$

where $C_{i}$ is nominal consumption in sector $i$. By construction $\sum_{i} c_{i}=1$. The calibrated model ${ }^{15}$ reproduces the observed nominal flow of goods. A general representation of output of

\footnotetext{
${ }^{15}$ We eliminate all sectors in the data set that do not produce (e.g. Coke, Refined Petroleum and Nuclear Fuel in Luxembourg). Moreover the aggregated final consumption sector includes also some negative values (e.g.
} 
the model is shown in table 2 . Where $p_{i} x_{i, j}$ is the equilibrium nominal flow of goods from sector

\begin{tabular}{|l|l|l|l|l|l|l|}
\hline sectors & sector 1 & sector 2 & sector $n$ & value input & VA & value production \\
\hline sector 1 & $p_{1} x_{1,1}$ & $p_{2} x_{1,2}$ & $p_{n} x_{1, n}$ & $\sum_{j=1}^{n} p_{j} x_{1, j}$ & $l_{1} \eta$ & $p_{1} x_{1}$ \\
sector 2 & $p_{1} x_{2,1}$ & & & & & \\
sector $n$ & $p_{1} x_{n, 1}$ & & & & & \\
\hline value intermediate & $\sum_{i=1}^{n} p_{1} x_{i, 1}$ & & & & & \\
\hline final consumption & $p_{1} d_{1}$ & & & & \\
\hline value use & $p_{1}\left(d_{1}+\sum_{i=1}^{n} p_{1} x_{i, 1}\right)$ & & & & & \\
\hline
\end{tabular}

Table 2: Input-output table from the model

$j$ to sector $i\left(p_{i}\right.$ is the equilibrium price of $\operatorname{good} i$ and $x_{i, j}$ is the equilibrium quantity of good $j$ used by sector $i$ ). The value of final consumption of good $i$ is given by $p_{i} d_{i}$, where $d_{i}$ is the demand for goods $i$ by the household. Total nominal production is given by $p_{i} x_{i}$. The labor hired by sector $i$ is $l_{i}$. The model uses nominal values to determine prices and quantities.

\section{References}

Acemoglu, D., Akcigit, U., Kerr, W., et al. (2015). Networks and the macroeconomy: An empirical exploration. NBER Chapters.

Acemoglu, D., Carvalho, V. M., Ozdaglar, A., and Tahbaz-Salehi, A. (2012). The network origins of aggregate fluctuations. Econometrica, 80(5):1977-2016.

Bonacich, P. (1972). Technique for analyzing overlapping memberships. Sociological methodology, 4:176-185.

Bonacich, P. and Lloyd, P. (2001). Eigenvector-like measures of centrality for asymmetric relations. Social Networks, 23(3):191-201.

Brin, S. and Page, L. (1998). The anatomy of a large-scale hypertextual web search engine. Computer networks and ISDN systems, 30(1):107-117.

Carvalho, V. M. (2014). From micro to macro via production networks. The Journal of Economic Perspectives, pages 23-47.

Chinazzi, M., Fagiolo, G., Reyes, J. A., and Schiavo, S. (2013). Post-mortem examination of the international financial network. Journal of Economic Dynamics and Control, 37(8):16921713

changes in inventories and valuables final consumption sector), therefore the sum of all final consumption sectors can be negative. Since the model does not allow for savings, we set negative values of $D_{i}$ to zero. 
Clauset, A., Shalizi, C. R., and Newman, M. E. (2009). Power-law distributions in empirical data. SIAM review, 51(4):661-703.

Haldane, A. G. et al. (2009). Rethinking the financial network. Speech delivered at the Financial Student Association, Amsterdam, April, pages 1-26.

Horvath, M. (1998). Cyclicality and sectoral linkages: Aggregate fluctuations from independent sectoral shocks. Review of Economic Dynamics, 1(4):781-808.

Horvath, M. (2000). Sectoral shocks and aggregate fluctuations. Journal of MOnetary Economics, (45):69-106.

Kali, R. and Reyes, J. (2007). The architecture of globalization: a network approach to international economic integration. Journal of International Business Studies, 38(4):595-620.

Kali, R. and Reyes, J. (2010). Financial contagion on the international trade network. Economic Inquiry, 48(4):1072-1101.

Katz, L. (1953). A new status index derived from sociometric analysis. Psychometrika, 18(1):3943.

Kleinberg, J. M. (1999). Authoritative sources in a hyperlinked environment. Journal of the $A C M(J A C M), 46(5): 604-632$.

Long Jr, J. B. and Plosser, C. I. (1983). Real business cycles. The Journal of Political Economy, pages 39-69.

Perra, N. and Fortunato, S. (2008). Spectral centrality measures in complex networks. Physical Review E, 78(3):036107.

Schiavo, S., Reyes, J., and Fagiolo, G. (2010). International trade and financial integration: a weighted network analysis. Quantitative Finance, 10(4):389-399.

Timmer, M., Erumban, A. A., Gouma, R., Los, B., Temurshoev, U., de Vries, G. J., Arto, I.-a., Genty, V. A. A., Neuwahl, F., Francois, J., et al. (2012). The world input-output database (wiod): contents, sources and methods. Technical report, Institute for International and Development Economics. 
1. L. Colombo, H. Dawid, Strategic Location Choice under Dynamic Oligopolistic Competition and Spillovers, Novembre 2013.

2. M. Bordignon, M. Gamalerio, G. Turati, Decentralization, Vertical Fiscal Imbalance, and Political Selection, Novembre 2013.

3. M. Guerini, Is the Friedman Rule Stabilizing? Some Unpleasant Results in a Heterogeneous Expectations Framework, Novembre 2013.

4. E. Brenna, C. Di Novi, Is caring for elderly parents detrimental to women's mental health? The influence of the European North-South gradient, Novembre 2013.

5. F. Sobbrio, Citizen-Editors' Endogenous Information Acquisition and News Accuracy, Novembre 2013.

6. P. Bingley, L. Cappellari, Correlation of Brothers Earnings and Intergenerational Transmission, Novembre 2013.

7. T. Assenza, W. A. Brock, C. H. Hommes, Animal Spirits, Heterogeneous Expectations and the Emergence of Booms and Busts, Dicembre 2013.

8. D. Parisi, Is There Room for 'Fear' as a Human Passion in the Work by Adam Smith?, Gennaio 2014.

9. E. Brenna, F. Spandonaro, Does federalism induce patients' mobility across regions? Evidence from the Italian experience, Febbraio 2014.

10. A. Monticini, F. Ravazzolo, Forecasting the intraday market price of money, Febbraio 2014.

11. Tiziana Assenza, Jakob Grazzini, Cars Hommes, Domenico Massaro, $P Q$ Strategies in Monopolistic Competition: Some Insights from the Lab, Marzo 2014.

12. R. Davidson, A. Monticini, Heteroskedasticity-and-Autocorrelation-Consistent Bootstrapping, Marzo 2014.

13. C. Lucifora, S. Moriconi, Policy Myopia and Labour Market Institutions, Giugno 2014.

14. N. Pecora, A. Spelta, Shareholding Network in the Euro Area Banking Market, Giugno 2014.

15. G. Mazzolini, The economic consequences of accidents at work, Giugno 2014.

16. M. Ambrosanio, P. Balduzzi, M. Bordignon, Economic crisis and fiscal federalism in Italy, Settembre 2014.

17. P. Bingley, L. Cappellari, K. Tatsiramos, Family, Community and Long-Term Earnings Inequality, Ottobre 2014.

18. S. Frazzoni, M. L. Mancusi, Z. Rotondi, M. Sobrero, A. Vezzulli, Innovation and export in SMEs: the role of relationship banking, Novembre 2014.

19. H. Gnutzmann, Price Discrimination in Asymmetric Industries: Implications for Competition and Welfare, Novembre 2014.

20. A. Baglioni, A. Boitani, M. Bordignon, Labor mobility and fiscal policy in a currency union, Novembre 2014.

21. C. Nielsen, Rational Overconfidence and Social Security, Dicembre 2014.

22. M. Kurz, M. Motolese, G. Piccillo, H. Wu, Monetary Policy with Diverse Private Expectations, Febbraio 2015.

23. S. Piccolo, P. Tedeschi, G. Ursino, How Limiting Deceptive Practices Harms Consumers, Maggio 2015.

24. A.K.S. Chand, S. Currarini, G. Ursino, Cheap Talk with Correlated Signals, Maggio 2015. 
25. S. Piccolo, P. Tedeschi, G. Ursino, Deceptive Advertising with Rational Buyers, Giugno 2015.

26. S. Piccolo, E. Tarantino, G. Ursino, The Value of Transparency in Multidivisional Firms, Giugno 2015.

27. G. Ursino, Supply Chain Control: a Theory of Vertical Integration, Giugno 2015.

28. I. Aldasoro, D. Delli Gatti, E. Faia, Bank Networks: Contagion, Systemic Risk and Prudential Policy, Luglio 2015.

29. S. Moriconi, G. Peri, Country-Specific Preferences and Employment Rates in Europe, Settembre 2015.

30. R. Crinò, L. Ogliari, Financial Frictions, Product Quality, and International Trade, Settembre 2015.

31. J. Grazzini, A. Spelta, An empirical analysis of the global input-output network and its evolution, Ottobre 2015. 